\title{
A REVIEW OF EVIDENCE ON THE COST-EFFECTIVENESS OF LOCAL AUTHORITY ACTIVITIES TO REDUCE EXPOSURE TO AIR POLLUTION FROM ROAD TRAFFIC
}

\author{
JO BARNES \& BEN WILLIAMS \\ Air Quality Management Resource Centre, University of the West of England, UK
}

\begin{abstract}
This paper presents a systematic review of evidence of the cost-effectiveness of local authority activities to reduce exposure to air pollution from road traffic. The work was undertaken as part of an evidence review for the National Institute for Health and Care Excellence (NICE) and contributes to the economic model produced by Eunomia, which underpins draft guidance for local government published by the Public Health and Social Care Centre (PHSCC) within NICE. The draft guidance was released for consultation in December 2016 and the final publication is expected in July 2017. The review addresses the scope outlined by the PHSCC and was undertaken following NICE search protocols with PHSCC approval. The scope covers interventions that could be delivered by local authorities to reduce road-traffic-related emissions by: reducing overall mileage; altering the type of fuel used or driving style; aiding dispersion or deposition of pollutants; and altering personal behaviour to reduce exposure to pollutants. This paper concludes that there is a lack of available published evidence on the cost-effectiveness of such measures and makes recommendations for further research to address this evidence gap.
\end{abstract}

Keywords: cost-effectiveness, NICE, local government, air pollution, road traffic, mitigation, public health, guidance, evidence review, systematic review.

\section{INTRODUCTION}

Air pollution from road traffic is a significant public health problem, accounting for $34 \%$ of nitrogen oxides (NOx), 14\% of $\mathrm{PM}_{10}$ and $13 \%$ of $\mathrm{PM}_{2.5}$ emissions in the UK in 2015 [1]. $\left(\mathrm{PM}_{10}\right.$ refers to particulate matter up to 10 microns; $\mathrm{PM}_{2.5}$ refers to particulate matter up to 2.5 microns.) In a report published in February 2016, the Royal College of Physicians and the Royal College of Paediatrics and Child Health [2] estimated that an estimated 40,000 premature deaths in the UK each year are attributable to outdoor air pollution.

In the UK, local authorities have a responsibility to manage air quality in their jurisdictions and to implement Air Quality Action Plans (AQAPs) where ambient (outdoor) concentrations of pollutants exceed health-based thresholds. These AQAPs and AQAP Progress Reports are reported to the Department for the Environment, Food and Rural Affairs (Defra) and are used to support the UK's compliance reporting against the EU Ambient Air Quality Directive (2008/50/EC).

In 2015, the National Institute for Health and Care Excellence (NICE) [3] began preparing public health guidance for local government on "Air pollution: outdoor air quality and health" under referral from the Department of Health in England. NICE is an independent organisation responsible for providing national guidance on promoting good health and preventing and treating disease. The guidance is intended for local authority staff working in public health, including environmental health, transport, planning and local air quality management, but may also be relevant for healthcare professionals in primary and secondary care; local government elected members; employers in all sectors (including transport operators), local enterprise partnerships members, and local businesses and developers; 
people working in the voluntary sector and non-governmental organisations, education and the general public.

In preparation of the guidance, the Public Health and Social Care Centre (PHSCC) within NICE undertook a review of published evidence on the effectiveness and cost-effectiveness of local authority interventions to reduce traffic related air pollution. In December 2015, NICE commissioned research to complement their evidence review on the cost-effectiveness of these interventions and to develop an economic model [4]. This paper presents the systematic evidence review of the cost-effectiveness of interventions to reduce traffic related air pollution contributing to the development of the economic model.

\section{METHODOLOGY}

The focus of this review was to find evidence of the cost-effectiveness of interventions that may be delivered by local authorities to reduce road-traffic-related emissions by: reducing overall mileage; altering the type of fuel used or driving style; aiding dispersion or deposition of pollutants; and altering personal behaviour to reduce exposure to pollutants. The review was undertaken following NICE search protocols [5] with PHSCC approval. Following the scope outlined by the PHSCC [6], this included four key topics:

1. Environmental change and development planning:

- planning and land allocations, development control and planning decisions, urban space and building design

- developing public transport routes and services

- developing routes and infrastructure to support low emission modes of transport

- measures to promote absorption, adsorption or impingement deposition, and catalytic action including: natural and artificial barriers (such as trees and foliage), surface treatments (such as titanium oxides) and dust suppressants (such as calcium magnesium acetate).

2. Traffic management, enforcement, and financial incentives and disincentives:

- $\quad$ traffic management systems and signal coordination

- $\quad$ zoning, including low (and ultra-low) emission zones

- $\quad$ parking restrictions and charges

- $\quad$ vehicle 'idling' restriction and charges.

3. Initiatives providing information, advice, education or developing skills for:

- travel planning (personalised travel planning and settings-based planning, such as in workplaces or schools), including awareness raising and education to encourage people to use alternatives to a car

- fuel choice, including zero-emission vehicles

- $\quad$ driver training, for example how to avoid heavy acceleration or braking and excessive speed

- $\quad$ route choice.

4. Advice and warnings (such as forecasts, text alerts or emails) for the public and people at particular risk.

Under these four topics, a set of 12 Review Questions were posed by NICE (Table 1). 
Table 1: Review questions by topic.

\begin{tabular}{ll}
\hline Topic 1: Environmental change and development planning \\
\hline 1 & $\begin{array}{l}\text { Are planning development control decisions and interventions effective and cost- } \\
\text { effective at reducing the health impact of, or people's exposure to, traffic-related } \\
\text { air pollution? }\end{array}$ \\
\hline 2 & $\begin{array}{l}\text { Are interventions to develop public transport routes and services, effective and } \\
\text { cost-effective at reducing the health impact of, or people's exposure to, traffic- } \\
\text { related air pollution? }\end{array}$ \\
\hline $3 \quad \begin{array}{l}\text { Are interventions to develop routes and infrastructure to support low emission } \\
\text { modes of transport effective and cost-effective at reducing the health impact of, } \\
\text { or people's exposure to, traffic-related air pollution? }\end{array}$ \\
\hline 4 & $\begin{array}{l}\text { Are measures to promote absorption, adsorption or impingement deposition, and } \\
\text { catalytic action effective and cost-effective at reducing the health impact of, or } \\
\text { people's exposure to, traffic-related air pollution? }\end{array}$ \\
\hline
\end{tabular}

\begin{tabular}{ll}
\hline $\begin{array}{l}\text { Topic 2: Traffic management and enforcement, and financial incentives and } \\
\text { disincentives }\end{array}$ \\
\hline 5 & $\begin{array}{l}\text { Are traffic management systems and signal coordination interventions effective } \\
\text { and cost-effective at reducing the health impact of, or people's exposure to, } \\
\text { traffic-related air pollution? }\end{array}$ \\
\hline 6 & $\begin{array}{l}\text { Are zoning interventions effective and cost-effective at reducing the health } \\
\text { impact of, or people's exposure to, traffic-related air pollution? }\end{array}$ \\
\hline 7 & $\begin{array}{l}\text { Are parking restrictions and charges effective and cost-effective at reducing the } \\
\text { health impact of, or people's exposure to, traffic-related air pollution? }\end{array}$ \\
\hline 8 & $\begin{array}{l}\text { Are vehicle 'idling' restrictions and charges, including waiting and loading } \\
\text { restrictions, effective and cost-effective at reducing the health impact of, or } \\
\text { people's exposure to, traffic-related air pollution? }\end{array}$
\end{tabular}

Topic 3: Travel Planning and other initiatives providing information, advice, education and skill development

$9 \quad$ Are settings-based travel planning (such as in workplaces, new residential developments or schools) interventions effective and cost-effective at reducing the health impact of, or people's exposure to, traffic-related air pollution?

10 Are personalised travel planning interventions to support low emission travel choices effective and cost-effective at reducing the health impact of, or people's exposure to, traffic-related air pollution?

11 Are driver information, education and training interventions effective and costeffective at reducing the health impact of, or people's exposure to, traffic-related air pollution?

Topic 4: Advice and warnings for the public and people at particular risk:

12 Are interventions providing advice and warnings for the public and people at particular risk effective and cost-effective at reducing the health impact of, or people's exposure to, traffic-related air pollution? 


\subsection{Search protocol}

In order to effectively build on the initial evidence review undertaken by the NICE team, the same search protocol (with minor modification, i.e. dates) was followed, but with the use of additional databases, locations and search terms [5]. A number of elements within the protocol were common across each question namely:

- searches;

- types of study to be included/excluded;

- participants/population;

- methods for selecting evidence (data screening);

- data extraction and quality assessment;

- $\quad$ strategy for data synthesis;

- analysis of subgroups;

- any other information or criteria for inclusion or exclusion.

\subsection{Search databases}

A review of available search databases and database providers, in consultation with the NICE Information Services team, concluded that the following databases should be included:

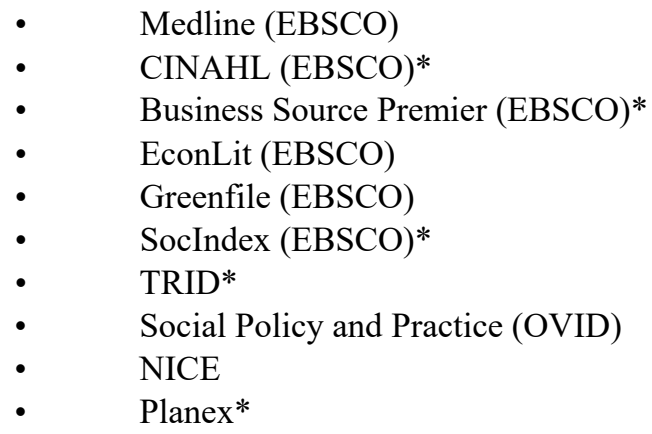

Asterisked (*) databases were additional to those used in the initial NICE evidence review. The inclusion of databases already used by NICE was justified on the basis of the use of additional search terms and the inclusion of the most recent publications.

\subsection{Search terms}

Search terms were developed and agreed with NICE for categories relating to each Review Question (RQ), 'Traffic', 'Air Pollution', 'Health' and 'Cost'. Search terms under these categories were considered necessary to identify evidence specific to the cost-effectiveness of interventions delivered by local authorities to reduce road-traffic-related emissions.

Although six of the databases (MEDLINE, CINAHL, Greenfile, EconLit, Business Source Premier and SocINDEX) were accessed via the same provider (EBSCO), in order to maximise the search functionality of each of the databases, searches were conducted for each separately. These databases also facilitated use of additional index search terms. These are detailed in Table 3.

For other databases (TRID, Social Policy and Practice, NICE and Planex), limited search functionality restricted the number of search terms that could be used, with the latter three limited to search terms that were not Review Question specific. 
Table 2: Search terms by category.

\begin{tabular}{|c|c|}
\hline Cate & earch terms \\
\hline Traffic & $\begin{array}{l}\text { (bus OR buses OR car OR cars OR HDV OR "heavy duty vehicle*" OR HGV OR } \\
\text { "heavy goods vehicle*" OR LGV OR "light goods vehicle*" OR LDV OR "light duty } \\
\text { vehicle*" OR lorry OR lorries OR "motor vehicle*" OR motorbike* OR motorcycle* } \\
\text { OR taxi OR taxis OR fleet OR van OR vans OR automobile* OR truck OR road* OR } \\
\text { highway* OR motorway* OR "rush hour" OR rush-hour OR street* OR "tail back*" } \\
\text { OR tail-back* OR tailback* OR traffic OR congestion OR transport* OR "trunk } \\
\text { route*" OR idling OR "vehicle parc" OR pedestrian* OR cyclist* OR driver* OR } \\
\text { driving OR commute*) }\end{array}$ \\
\hline $\begin{array}{l}\text { Air } \\
\text { Pollution }\end{array}$ & 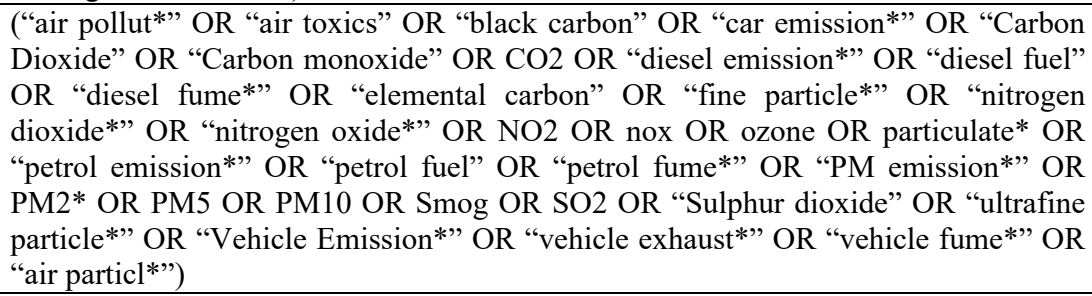 \\
\hline Health & $\begin{array}{l}\text { ("Inhalation Exposure" OR "inhalation exposure" OR Cardiovascular OR CVD OR } \\
\text { Disease OR Health OR Mortality OR Threshold* OR Stroke OR Asthma OR "Blood } \\
\text { pressure" OR "Body mass index" OR BMI OR Cancer OR "Heart disease" OR } \\
\text { "Physical health" OR "Mental health" OR Diabetes OR "Heat vulnerability" OR } \\
\text { "Health equity" OR "Health inequality" OR COPD OR "Chronic Obstructive } \\
\text { Pulmonary Disease" OR "premature deaths" OR epidemiology OR "Child* ill health" } \\
\text { OR "Vulnerable population" OR "Elderly Ill health" OR Elderly OR "Ill-health" OR } \\
\text { "Hospital admissions for respiratory disease" OR "GP attendance" OR Morbidity OR } \\
\text { "Quality Adjusted Life Years" OR "Disability Adjusted Life Years" OR QALY OR } \\
\text { DALY OR exposure OR Death* OR "child mortality" OR "health outcome" OR } \\
\text { respiratory OR ischaemic OR "birth defects" OR "low birth weight" OR "congenital } \\
\text { anomalies" OR "years of life lost" OR "lung function" OR cerebrovascular OR } \\
\text { myocardial OR "heart attack" OR "oxidative stress" OR vascular OR "premature birth" } \\
\text { OR "preterm birth" OR "pre-term birth") }\end{array}$ \\
\hline Cost & $\begin{array}{l}\text { (budget* OR CBA OR CCA OR Cost* OR CUA OR economic OR Expenditure OR } \\
\text { financ* OR fund* OR Investment OR "net benefit" OR Value OR Revenue) }\end{array}$ \\
\hline RQ1 & $\begin{array}{l}\text { (city OR cities OR town* OR urban OR building* OR environment*) N2 (plan* OR } \\
\text { develop* OR design* OR infrastructure) }\end{array}$ \\
\hline$\overline{\mathrm{RQ} 2}$ & $\begin{array}{l}\text { (bus OR metro OR vehicle* OR rail OR train OR trains OR tram OR trams OR } \\
\text { trolleybus* OR tube OR underground OR psv) N2 (lane OR lanes OR route* OR } \\
\text { service* OR trip OR trips OR line OR lines) OR (bus OR metro OR vehicle* OR rail } \\
\text { OR train OR trains OR tram OR trams OR trolleybus* OR tube OR underground OR } \\
\text { psv) N2 (lane OR lanes OR route* OR service* OR trip OR trips OR line OR lines) }\end{array}$ \\
\hline RQ3 & $\begin{array}{l}\text { (low OR zero OR standard*) N1 (carbon OR emission* OR ultra) OR ("EURO 3" OR } \\
\text { "EURO 4" OR "EURO 5" OR "EURO 6" OR "EURO III" OR "EURO IV" OR } \\
\text { "EURO VI" OR "EURO standard") OR (electric* OR hybrid* OR green OR clean OR } \\
\text { sustainab*) AND (vehicle* OR car* OR bus* OR taxi* OR transport OR technolog*) } \\
\text { OR (cycle OR bicycle OR bike OR cycling OR cyclist*) N1 (corral* OR lane* OR } \\
\text { rental* OR route* OR sharing* OR share) }\end{array}$ \\
\hline
\end{tabular}


Table 2: Continued.

\begin{tabular}{|c|c|}
\hline Category & earch terms \\
\hline RQ4 & $\begin{array}{l}\text { ("urban greening" OR vegetation OR hedge* OR plants OR planting OR tree* OR } \\
\text { foliage OR "urban woodland*" OR "ecological engineering" OR ecosystem*) OR } \\
\text { (dispersion OR deposition OR absorption OR adsorption OR impingement OR } \\
\text { barrier*) N3 (road* OR street* OR kerb* OR pavement* OR highway* OR motorway* } \\
\text { OR intersection* OR traffic OR vehicle*) OR (green N2 (wall* OR roof* OR rooves } \\
\text { OR infrastructure OR space*)) OR ("road surface*" OR "dust suppressant*" OR } \\
\text { "porous asphalt" OR "very open asphalt" OR "calcium magnesium acetate" OR } \\
\text { "surface treatment*" OR "titanium oxide*" OR "titanium dioxide*") OR ("catalytic } \\
\text { action" OR photocataly*) and (pavement* OR paving OR concrete OR asphalt OR } \\
\text { surface* OR road* OR highway* OR street*) }\end{array}$ \\
\hline RQ5 & $\begin{array}{l}\text { (traffic N2 (ban OR bans OR calm* OR flow* OR integrat* OR restrict* OR } \\
\text { management OR light* OR signal* OR system* OR continuous)) OR (road N2 } \\
\text { (intersection* OR junction* OR marking* OR sign OR signs)) OR ("emission* testing" } \\
\text { OR "green wave" OR "lane control") }\end{array}$ \\
\hline RQ6 & $\begin{array}{l}\text { ((vehicle* OR traffic OR speed* OR parking) N2 (ban OR bans OR banned OR calm* } \\
\text { OR charg* OR control* OR enforce* OR fine OR fines OR fined OR flow* OR } \\
\text { manage* OR pay OR pays OR payment OR paid OR reduce* OR reduction* OR } \\
\text { restrict* OR limit*)) OR (("20mph" OR " } 20 \text { mph" OR home OR "low emission") N2 } \\
\text { (zone* OR zoning)) OR (LEZ OR ULEZ) OR (Street* N2 (closure* OR safe OR safer } \\
\text { OR pedestrian*)) }\end{array}$ \\
\hline RQ7 & $\begin{array}{l}\text { (Parking N2 (car OR charg* OR fine* OR control* OR enforce* OR multistorey OR } \\
\text { multi-storey OR restrict* OR zone* OR zoning OR resident*) OR (RPZ*)) }\end{array}$ \\
\hline RQ8 & $\begin{array}{l}\text { ((Vehicle OR engine) N2 (control* OR enforce* OR idling OR loading OR restrict* } \\
\text { OR waiting OR switch off OR switchoff OR switch-off)) OR (idling OR waiting OR } \\
\text { loading) N2 (zone OR zoning OR charg*) }\end{array}$ \\
\hline RQ9 & $\begin{array}{l}\text { ((Plan* N1 (travel* OR journey*)) AND (residential OR school* OR workplace* OR } \\
\text { development*)) OR (((Active N2 (transport* OR travel*)) OR (*cycl* OR *bike* OR } \\
\text { pedestrian* OR Rail OR train OR tram OR walk*) AND (Willingness OR commut* } \\
\text { OR "school run" OR "modal shift"))) OR (Car N1 (club* OR dependence OR pool* } \\
\text { OR share OR sharing OR Lite OR lift OR passenger* OR city)) OR (((Vehicle* OR } \\
\text { transport*) N2 (journey* OR trip* OR use*)) AND (behaviour OR behavior OR mode } \\
\text { OR modal OR occupancy) AND (change* OR choice* OR shift)) }\end{array}$ \\
\hline RQ10 & $\begin{array}{l}\text { ((Plan* AND (travel* OR journey*)) AND (personal*OR individual*) OR (PTP)) OR } \\
((\text { Active N2 (transport* OR travel*)) OR ((*cycl* OR *bike* OR pedestrian* OR Rail } \\
\text { OR train OR tram OR walk* OR bus OR buses OR "public transport") AND (Willing* } \\
\text { OR commut* OR "school run" OR "modal shift"))) OR (Car N1 (club* OR dependence } \\
\text { OR pool* OR share OR sharing OR Lite OR lift OR passenger OR occupancy)) OR } \\
(((\text { Vehicle* OR transport*) N2 (journey* OR trip* OR use*)) AND (behaviour OR } \\
\text { behavior OR mode OR modal OR occupancy) AND (change* OR choice*)) }\end{array}$ \\
\hline RQ11 & $\begin{array}{l}\text { ((driver* OR driving) AND (aware* OR behavior* OR behaviour* OR choice* OR } \\
\text { educat* OR habit* OR inform* OR style* OR pattern* OR training OR eco OR } \\
\text { initiative* OR intervention*)) OR ((Acceleration OR braking OR deceleration OR } \\
\text { "stop go" OR stop-go OR "stop start" OR mile* OR consumption OR economy OR } \\
\text { route*) AND (advice OR advise OR inform* OR educat* OR training OR initiative* } \\
\text { OR intervention*)) OR (("alternative fuel*" OR biodiesel* OR biofuel* OR CNG OR } \\
\text { "compressed natural gas" OR electric* OR hybrid OR "liquefied petroleum gas" OR } \\
\text { "liquid petroleum gas" OR "liquified petroleum gas" OR "low carbon*" OR LPG OR } \\
\text { plugged-in) AND (advice OR advise OR inform* OR educat* OR training OR } \\
\text { initiative* OR intervention*)) OR (("ECO Stars" OR ECOStars OR FORS) AND } \\
\text { (freight OR fleet)) }\end{array}$ \\
\hline
\end{tabular}


Table 2: Continued.

\begin{tabular}{|l|l|}
\hline Category & Search terms \\
\hline RQ12 & $\begin{array}{l}\text { ((Public OR vulnerable OR at-risk) AND (advice OR advisory OR Advocacy OR } \\
\text { Communicat* OR "exposure risk*” OR forecast* OR warning*) AND (audiovisual OR } \\
\text { audio-visual OR display OR email OR Hotlines OR media OR SMS OR telephone OR } \\
\text { phone OR telehealth OR text* OR voice-mail* OR voicemail* OR video* OR "social } \\
\text { media" OR Facebook OR Twitter OR RSS)) OR (“Air quality index" OR "Air quality } \\
\text { indices" OR airtext OR airAlert OR "AQ index" OR "AQ indices") }\end{array}$ \\
\hline
\end{tabular}

Table 3: Additional index search terms by category.

\begin{tabular}{|c|c|}
\hline Category & Additional index search terms \\
\hline Cost & $\begin{array}{l}\text { (MH "Costs and Cost Analysis+") OR (MH "Cost of Illness") OR (MH "Cost-Benefit } \\
\text { Analysis") OR (MH "Health Care Costs+") OR (MH "Economics+") OR (MH "Budgets+") }\end{array}$ \\
\hline $\begin{array}{l}\text { Air } \\
\text { Pollution }\end{array}$ & $\begin{array}{l}\text { (MH “Air Pollution+") OR (MH “Air Pollutants+") OR (MH "Smog") OR (MH "Inhalation } \\
\text { Exposure") OR (MH "Particulate Matter+") OR (MH “Carbon Dioxide") OR (MH “Carbon } \\
\text { Monoxide") OR (MH "Nitrogen Oxides") OR (MH "Nitrogen Dioxide") OR (MH "Ozone") } \\
\text { OR (MH "Vehicle Emissions") }\end{array}$ \\
\hline Transport & $\begin{array}{l}\text { (MH “Motor Vehicles") OR (MH “Automobiles") OR (MH “Automobile Driving") OR } \\
\text { (MH “Transportation") }\end{array}$ \\
\hline RQ1 & $\begin{array}{l}\text { (MH “City Planning”) OR (MH “Social Planning”) OR (MH “Environment Design”) OR } \\
\text { (MH "Urban Renewal") }\end{array}$ \\
\hline RQ3 & (MH “Bicycling”) \\
\hline RQ4 & (MH “Parks, Recreational”) \\
\hline RQ7 & (MH “Parking facilities") \\
\hline RQ8 & (MH "vehicle Operation") \\
\hline RQ9 & (MH bicycles) \\
\hline RQ11 & (MH "Natural Gas") \\
\hline RQ12 & xt messaging \\
\hline
\end{tabular}

Where search functionality permitted, Title/Abstract searches were conducted for the categories 'Traffic', 'Air Pollution', 'Health' and 'Cost' collectively combined with each of the Review Question search terms. Results were filtered for peer reviewed articles, published in English between 1996 and 2016, where possible and then exported as ris files. Planex results were not able to be exported as ris files and these were exported to email. The results of NICE's initial evidence review were also supplied as a ris file to enable deduping of the new results.

\subsection{Additional sources}

In addition to the databases searches, 111 Local Authority Air Quality Action Plans and Action Plan Progress Reports were also reviewed. These were obtained from the Defra AQMA website [7] and selected on the basis of those that had included cost information for the proposed measures.

Finally, additional grey literature on effectiveness and financial costs of interventions were reviewed from a range of sources, including:

- Defra;

- Sustrans;

- Living Streets;

- Streets Alive;

- Forest Research; 
- Playing Out;

- WHO;

- World Bank;

- REVIHAAP;

- HRAPIE;

- Public health outcomes framework; and

- ECO Stars.

\section{ANALYSIS}

The exported ris files were imported to the Mendeley reference manager (https://www.mendeley.com/) and tagged according to their source database. Mendeley automatically detects duplicate files and stores them as one; thereby records from more than one source are automatically tagged with all relevant source tags. Records were then able to be selected by tag and removed from the parent folder (while retaining in All Documents). In this way, the records obtained from NICE from their initial evidence review could be tagged and removed from the records downloaded from the databases above. This is a novel approach to deduping records that the authors had not observed elsewhere.

All remaining deduped results were searched for ' $U K$ ' in Mendeley to narrow the scope of records to be reviewed. Titles and abstracts were reviewed for relevance against all Review Questions, particularly selecting for records that included quantification (in terms of air quality, health or cost) of measures implemented to reduce traffic-related air pollution, preferably in a UK or EU context. Once the 'UK' search results were reviewed the remaining titles were skimmed for relevance. All relevant records were flagged as 'favourites' in Mendeley and, where available, full text articles were reviewed and if appropriate downloaded. Planex records were also reviewed separately.

\section{RESULTS}

The literature review identified 6556 records, including peer-reviewed articles, conference proceedings and grey literature, of which 4543 were additional to those provided by NICE from their initial evidence review. Of these, only 138 records were identified as potentially relevant, with 57 full texts downloaded for further investigation (Table 4 -note, some records were identified in multiple databases).

Greenfile generated the greatest number of search results, followed by Medline, TRID, CINAHL and Business Source Premier, the latter three of which were databases previously not included in NICE's search strategy. In terms of potentially relevant records, however, TRID, NICE and Greenfile were the most productive. Most records found in TRID, NICE or Social Policy and Practice were unique to those databases, but there was more overlap between the other databases, perhaps unsurprisingly as these are all provided by EBSCO.

Very few downloaded records were specific to any one RQ. The largest number of RQ-specific results were attributable to RQ3 (Interventions to develop routes and infrastructure to support low emission modes of transport) and focussed on active travel (waking and cycling). There were also a few results relating to RQ6 (Zoning interventions), but the majority of records were either relevant across multiple RQs or were potentially useful but not RQ-specific (Miscellaneous) (Table 5). No records specific to RQ5 (Traffic management systems and signal coordination interventions), RQ8 (Vehicle 'idling' restrictions and charges, including waiting and loading restrictions), RQ10 (Personalised travel planning interventions to support low emission travel choices) or RQ11 (Driver information, education and training interventions) were identified. 
Table 4: Number of records by database.

\begin{tabular}{lccc}
\hline Database & $\begin{array}{c}\text { No. of records } \\
\text { (incl. previously } \\
\text { identified by NICE) }\end{array}$ & $\begin{array}{c}\text { No. of records } \\
\text { (excl. previously } \\
\text { identified by NICE) }\end{array}$ & $\begin{array}{c}\text { No. of potentially } \\
\text { relevant records }\end{array}$ \\
\hline BSP & 713 & 639 & 12 \\
\hline CINAHL & 935 & 888 & 13 \\
\hline ECONLIT & 363 & 279 & 3 \\
\hline GREENFILE & 1595 & 1224 & 27 \\
\hline MEDLINE & 1058 & 899 & 3 \\
\hline SOCINDEX & 229 & 221 & 31 \\
\hline NICE (UWE) & 515 & 513 & 6 \\
\hline SOCIAL POLICY & 80 & 57 & 0 \\
\hline AND PRACTICE & 1033 & 933 & 51 \\
\hline TRID & 35 & 35 & 3 \\
\hline PLANEX & & & 0 \\
\hline
\end{tabular}

Table 5: Number of downloaded records by Review Question (RQ).

\begin{tabular}{|c|c|c|}
\hline \multicolumn{2}{|c|}{ Topic 1: Environmental change and development planning } & \multirow{2}{*}{$\begin{array}{l}\text { No. of selected papers } \\
4\end{array}$} \\
\hline 1 & Planning development control decisions and interventions & \\
\hline 2 & Interventions to develop public transport routes and services & 1 \\
\hline 3 & $\begin{array}{l}\text { Interventions to develop routes and infrastructure to support } \\
\text { low emission modes of transport }\end{array}$ & 12 \\
\hline 4 & $\begin{array}{l}\text { Measures to promote absorption, adsorption or impingement } \\
\text { deposition, and catalytic action }\end{array}$ & 1 \\
\hline \multicolumn{3}{|c|}{$\begin{array}{l}\text { Topic 2: Traffic management and enforcement, and financial } \\
\text { incentives and disincentives }\end{array}$} \\
\hline 5 & $\begin{array}{l}\text { Traffic management systems and signal coordination } \\
\text { interventions }\end{array}$ & 0 \\
\hline 6 & Zoning interventions & 7 \\
\hline 7 & Parking restrictions and charges & 1 \\
\hline 8 & $\begin{array}{l}\text { Vehicle 'idling' restrictions and charges, including waiting } \\
\text { and loading restrictions }\end{array}$ & 0 \\
\hline \multicolumn{3}{|c|}{$\begin{array}{l}\text { Topic 3: Travel Planning and other initiatives providing } \\
\text { information, advice, education and skill development }\end{array}$} \\
\hline 9 & $\begin{array}{l}\text { Settings-based travel planning (such as in workplaces, new } \\
\text { residential developments or schools) interventions }\end{array}$ & 3 \\
\hline 10 & $\begin{array}{l}\text { Personalised travel planning interventions to support low } \\
\text { emission travel choices }\end{array}$ & 0 \\
\hline 11 & Driver information, education and training interventions & 0 \\
\hline \multicolumn{3}{|c|}{$\begin{array}{l}\text { Topic 4: Advice and warnings for the public and people at } \\
\text { particular risk: }\end{array}$} \\
\hline 12 & $\begin{array}{l}\text { Interventions providing advice and warnings for the public } \\
\text { and people at particular risk }\end{array}$ & 1 \\
\hline & Miscellaneous & 27 \\
\hline
\end{tabular}




\section{DISCUSSION}

The original review of evidence on effectiveness and cost-effectiveness of interventions, undertaken by NICE, shortlisted 45 studies identified by a systematic search of relevant databases and call for evidence from registered stakeholders [4]. However, the complimentary search process undertaken by Eunomia and UWE mostly identified published evidence on effectiveness of interventions that were already included in the evidence review originally undertaken by NICE.

A critical review of the identified studies revealed that the reported studies have limited applicability in the context of a local authority in the UK, mainly due to the various geographical, social, cultural and economic differences. Moreover, evidence on cost-effectiveness of interventions could not be identified [4].

In the absence of sufficient published cost-effectiveness evidence for the interventions considered in the evidence review, it was necessary to develop an economic model to assess the cost-effectiveness of local authority interventions to reduce traffic related air pollution in the UK In selecting relevant records, therefore, interpretation of the brief was broadened to consider records that provided any quantification of the impact of the intervention, whether on air quality, health or cost in order to enable the economic model to synthesise the data on costs and effectiveness from different sources to estimate the net cost-effectiveness of the interventions from a UK local authority perspective. Full details are provided in the economic model report [4].

\section{CONCLUSIONS}

The absence of available published evidence on the cost-effectiveness of interventions to reduce traffic-related pollution is clearly apparent from this study. This is symptomatic of the difficulties in quantifying the effectiveness of local authority actions in relation to air quality due to a multitude of confounding factors (e.g. meteorological variability, lack of reliable monitoring or modelling data, interventions not delivered in isolation). However, there is a need for local government to be able to demonstrate the effectiveness of implemented interventions on reducing air pollution to local Councillors and the populace as well as in their statutory reporting to national government for Local Air Quality Management. Furthermore, it is important for cost-effectiveness of measures to be evaluated to facilitate their uptake.

In order to fill this knowledge gap, it is therefore recommended that further research is required to provide robust analysis of local authority interventions to reduce traffic-related air pollution, which address, but are not limited to, the Review Questions included in this study. Local government must therefore be allocated resources with which to generate the necessary data (traffic counts, pollutant concentrations, public health records, cost of intervention, etc.) and a framework methodology developed to ensure consistency, comparability and wider applicability of the results.

\section{ACKNOWLEDGEMENTS}

This work was funded by the National Institute for Health and Care Excellence (NICE) as part of a contract with Eunomia Research \& Consulting Ltd. Our thanks go to the members of the NICE Public Health Advisory Committee and NICE Information Services for this project, who provided valuable input to the methodology and data.

\section{REFERENCES}

[1] Defra, Emissions of Air Pollutants in the UK, 1970 to 2015, Statistical Release 21 December 2016, National Statistics, Online. https:/www.gov.uk/government/uploads/ 
system/uploads/attachment_data/file/579200/Emissions airpollutants_statisticalrelease 2016 final.pdf. Accessed on: 29 Jan. 2017.

[2] Royal College of Physicians. Every breath we take: the lifelong impact of air pollution. Report of a working party. London: RCP, 2016. Online. https://www.rcplondon.ac.uk/ projects/outputs/every-breath-we-take-lifelong-impact-air-pollution. Accessed on: 29 Jan. 2017.

[3] NICE, Air pollution: outdoor air quality and health, Consultation, Online. https://www.nice.org.uk/guidance/indevelopment/gid-phg92. Accessed on: 29 Jan. 2017

[4] Ballinger, A., Chowdhury, T., Sherrington, C. \& Cole, G., Air pollution: Economic analysis, Main Report, $17^{\text {th }}$ November 2016, Online. https://www.nice.org.uk/ guidance/GID-PHG92/documents/economic-report. Accessed on: 29 Jan. 2017.

[5] NICE, Developing NICE guidelines: The manual, process and methods [PMG20], October 2014. Online. https://www.nice.org.uk/process/pmg20/chapter/introductionand-overview. Accessed on: 2 Nov. 2015.

[6] NICE, Guideline scope, Air pollution: Outdoor air quality and health, Final. Online. https://www.nice.org.uk/guidance/GID-PHG92/documents/air-pollution-outdoor-airquality-and-health-final-scope2. Accessed on: 2 Nov. 2015.

[7] Defra, List of Local Authorities with AQMAs. Online. https://uk-air.defra.gov. uk/aqma/list?la=all\&country=all\&pollutant=no2. Accessed on: 29 Jan. 2017. 